\title{
Background:
}

Women with a raised body mass index (BMI) have an increased risk of pregnancyrelated complications and adverse outcomes compared to women with a healthy BMI. (CMACE 2010). Body mass index is calculated by dividing a person's weight in kilograms by the square of their height in metres. For most adults, an ideal BMI is in the 18.5 to 24.9 range; between 25 and 29.9 a person is in the overweight range and between 30 and 39.9 a person is in the "obese" range. For the purposes of this study, a raised $\mathrm{BMI}$ is 30 and above.

There is evidence that midwives find the discussion of weight and diet and exercise difficult (Stotland et al. 2010). The findings of an Australian study (Schmied et al. 2011) supported this with participants (midwives and obstetricians) reporting difficulties in communication with pregnant obese women. Participants of this study also believed that there was a high level of intolerance of obese pregnant women among health professionals. There is concern that health professionals lack the skills and knowledge in how to communicate with obese women about their weight (Heslehurst et al. 2007; Schmied et al. 2011) and also how to get the balance right when discussing the risks of obesity with regard to the psychological impact on the women.

These difficulties are reflected in the evidence regarding the experiences of women with raised BMls in pregnancy. The authors of a study in the UK (Furber \& McGowan 2011b) found that participants felt humiliated and stigmatised and that interactions with health professionals and the general public reinforced their discomfort about their size. The stigmatisation of women with raised BMI can result in discrimination with the potential to lead to a culture of intolerance about women who do not fit the norm (Wray \& Deery 2008).

\section{Rationale for the study}

There remain difficulties in communication between midwives and women about raised $\mathrm{BMI}$.

\section{Methods}

The aim of the study was to explore women's experiences of having a raised BMI whilst pregnant and whether information from midwives and other health professionals had an impact on their pregnancies. A qualitative approach was taken as this was considered the most approach means to find out about women's experiences. Need theory re. methodological basis of study!!

The more detailed aims of the study were:

- To investigate women's knowledge of raised BMI and raised risk in pregnancy and birth.

- To discover what women remember about information given to them by midwives and other health professionals.

- To explore the psychological impact of being obese in pregnancy. 
- To make recommendations related to the professional support of women with raised $\mathrm{BMI}$ (30 and above).

\section{$\underline{\text { Inclusion criteria }}$}

The only inclusion criterion was that the woman should have a raised BMI ( 30 or more) and be pregnant. There were no exclusion criteria.

\section{Study design:}

This exploratory qualitative study took the form of interviews $(n=9)$ with one joint interview $(n=2)$ to gather detailed information from pregnant women with a raised $\mathrm{BMI}$ (30 or more).

The study received approval from the NRES Committee South West - Cornwall and Plymouth.

\section{Recruitment:}

All the women were recruited from a hospital in the south west of England. Eligible women (those with a raised BMI of 30 or more) were approached by the midwife researcher when they came to an antenatal hospital appointment. The midwife researcher discussed the study with them and gave them a participant information sheet if they were interested in taking part. Women who expressed an interest were contacted by the midwife researcher by phone on a later date to allow them time to consider the study. Out of 31 women who were approached, 11 took part in the study. Three declined at the time, three who were contacted by phone said they were too busy and one had had a termination due to fetal abnormalities. The remaining 13 were unable to be contacted by phone.

All interviews took place in the woman's home. The joint interview took place in a meeting room on the hospital site. The latter was to be a focus group, but only two women were able to attend on the day due to transportation problems of the third member.

\section{Data collection:}

Written consent was obtained from all women prior to being interviewed. The interviews took place between January and June 2016. They were audio recorded and lasted between nine minutes and 42 minutes. An interview framework was used, consisting of four broad questions (see table 1), but participants were encouraged to discuss anything they felt appropriate. As the interviews progressed, and areas of interest to the women were discussed, additional questions were introduced. Whilst the interviews were conducted on a one-to-one basis, the joint interview had the addition of one of the other team members. She was present to ensure field notes were taken and also to provide a more detached viewpoint about the discussions. The interviews were audiotaped and transcribed checked by the midwife researcher to ensure accuracy. Field notes were also taken at the time to help with the quality of the analysis. In addition, each interview was listened to 
shortly after completion and notes taken as an aide memoire and also as an early start to the analysis. Once data saturation was reached, no further women were interviewed.

\section{Data analysis:}

The coding was done manually, using Word and Excel to systematically develop a coding framework. Thematic analysis (Braun and Clark, 2006) was used to develop the codes into sub-themes and then final themes (see table 2). A selection of the transcripts was independently coded by one of the co-investigators to ensure interrater reliability. A second member of the team was also involved in the development of the themes to provide additional rigour to the findings.

\section{FINDINGS:}

Population:

Three women were primiparous and eight women multiparous and all were in their third trimester of pregnancy. They were aged between 19 and 38 and were all white British. Their BMls ranged from $31.2 \mathrm{~kg} / \mathrm{m}^{2}$ to $47.3 \mathrm{~kg} / \mathrm{m}^{2}$.

The following themes were developed from the interviews and focus group to describe women's experiences:

1. Vulnerable

2. Being a good mother

3. Information gap

\section{VULNERABLE}

The vulnerability of this group of women was demonstrated in their expressions of feelings of guilt and also feelings that they were being judged because of their weight. They were also candid in their understanding and acceptance - or otherwise - of words to describe their weight and they were able to describe their interactions with midwives and other health professionals. It became apparent that the majority of women had a long history of dieting and self-weighing which provided the context for much of their knowledge and beliefs.

\section{Guilt and embarrassment}

Some expressed embarrassment about having extra tests and others expressed guilt about their raised BMI. Some participants spoke about it in terms of "other people" get upset and are sensitive about their weight (participants 1 and 6) whilst others were able to identify these feelings about themselves:

“.. you just feel I guess a bit guilty because you put yourself at this risk". (participant 10)

"I think there is an element that probably for most people, their status as a pregnant woman with a raised BMI is to a certain extent sort of self-inflicted 
and you know the needing of additional care as simply as a result of that does, yeah, definitely made me feel guilty. (participant 11)

Some explained how they felt judged and criticised by their midwife or health professional. Some women appeared to be hyper aware of how suggestions or recommendations from midwives (eg regarding diet and exercise) may be misconstrued as criticism:

“..oh well you didn't lose weight before you fell pregnant or you're not trying to lose weight now, so you don't care about your baby.." (participant 2)

"The only thing that bothered me I think was that you know you are bigger but then they write on the scans they couldn't see very much due to raised BMI it kind of like, it made me feel like I am so fat they can't see what they are looking for sort of thing, but I know they have to write it," (participant 7)

However, not everyone felt judged by health professionals and there was recognition that the discussions about weight were a necessary part of midwifery care:

"I feel like I have had excellent care and that nothing that has happened to me or you know nothing about the way I have been treated has made me feel like there is any judgement. I am probably my own harshest critic as I imagine most women probably are," (participant 11)

\section{$\underline{\text { Use of language }}$}

All participants were all asked about the acceptability of the terms 'raised BMI' as well as 'obese'. All stated that raised BMI or Body Mass Index was an acceptable factual term and one that was understandable to them. The women said that health professionals had used 'raised BMl' when discussing their weight rather than obese.

"They use BMI because it is less offensive" (participant 3).

"I think it's a good term because it's [BMI's] a medical term and um it's less emotive than um obesity or you know even weight. You know some people you know get a bit upset if increased weight is talked about especially if they are pregnant. Emotions are running high anyway. Er so yes I think it's a good enough term." (participant 1)

Obese was considered unacceptable - personal (participant 7), degrading (participant 2) and one stated "I don't think that's a nice word to use" (participant 4). Only one participant (11) stated that obese was acceptable as it was a factual word. The majority of women did not perceive themselves as obese. One participant (8) described obese people as "massive, massive people" and most explained that although it might factually describe them they did not feel that the word was appropriate to them.

"I see somebody that's obese as somebody that doesn't care about their weight, in a way... And when you, when I hear the word obese, I have seen obese people and I don't look like obese people." (participant 2) 
Others did not appear so distressed about their raised BMI. One participant (3) did not regard BMI as particular relevant to her, "It doesn't really mean anything to me to be honest because everyone is different". Language played an important role in women's perception of themselves as participant 5 demonstrated, "I always knew that I had a higher BMI because I'm, I have always been quite a heavy person, but I don't think that I am fat."

\section{$\underline{\text { Relationship with their midwife }}$}

The relationship with their midwives was discussed. Most participants appeared to have a good relationship with their midwives and stressed the support they had had during their pregnancy. This was not universally the case, with one participant (2) criticising the attitude of her midwife to her weight as very unhelpful and upsetting. A small number of women ( 6 and 3 ) felt their midwife had been embarrassed to address their weight and they felt the midwife had found the conversation difficult.

"I think they skirt around it. I think they are a bit scared to say it perhaps" (participant 6)

Others had found their midwife to be honest and professional about their weight and had presented it in a factual and non-judgemental way, as participant 8 explained: "I never felt labelled as a pregnant lady with a raised BMI, it was always just a 'well this is what the science shows".

One participant (2) feared bullying about her weight from her midwife, and indeed, had an unsatisfactory relationship with her. Another could foresee the potential problem of being judged and had a fear by some that midwives might be unsympathetic and insensitive, "You know the last thing you want is um to be given a lecture or anything like that," (participant 1).

\section{Long history of dieting}

"Weight has always been a huge thing for me, throughout my whole life ... I would love to be skinny, I would love to be thin, but it's hard" (participant 6)

The majority of the participants had a long history of dieting and trying to control their weight. Many had been in diet clubs, such as Weight Watchers and Slimming World and the participants were very body conscious and aware of their diet They were all aware of the importance of a healthy lifestyle, which included (in particular) not over eating, much of which possibly relates to their membership of diet clubs.

"I have always battled with my weight, I have always constantly battled, um, and I think she [midwife] knew that, she didn't really say a lot, um, obviously it bothers me, obviously I can't lose weight in pregnancy, I mean, I can eat healthily" (participant 7).

Only one participant spoke about her mental health history, although this was not an area explored in the interviews, 
"I'm trying not to worry about my weight too much at the end of the day because I do get obsessive about it and I have starved myself in the past and I am not doing it while I am pregnant" (participant 2)

\section{Being weighed}

The participants were asked about being weighed and how they found this experience. Most were used to being weighed - from their diet clubs - but many also spoke of regular self-weighing, with one participant weighing herself every day. Some women explained that to be weighed was a straightforward experience, albeit somewhat difficult:

"I think its fine to be weighed. I mean it wasn't great (laughter). No one likes being weighed but to be fair, I was doing slimming world so I was used to being weighed every week anyway," (participant 6)

"It's always embarrassing to stand on the scales, yeah, it was difficult, to be overweight before you've even, you know, started but it wasn't an issue at all from her, it's more me." (participant 10)

Participant 11 recalled that it was a 'factual' exercise which did not give her too much concern. Another said that she "hated it" (participant 2) and one participant had anticipated being weighed and explained she was worried about attending for this reason and its implications (participant 10). Participant 9 gave an indication of the difficulty she had being weighed:

"It's alright, it's sort of always, you always try and take off your heavy boots and stuff like that but it's fine".

\section{BEING A GOOD MOTHER}

This theme developed from the willingness of women to follow hospital guidelines and take responsibility for ensuring they did all they could to have a healthy pregnancy.

\section{Following guidelines}

The result of having a raised BMI whilst pregnant was being put on a different antenatal pathway involving additional interventions and appointments and women being categorised as high risk. The participants appeared happy to follow the recommendations of the midwives despite not having a full understanding of the reasons behind the invitation:

"Were reasons given? No, not particularly. I just did it to be honest, I didn't really question it. No, I don't really know why" (participant 3)

Whilst the participants' knowledge of the guidelines was very limited (which is discussed in the theme which follows) they appeared to trust their midwife to ensure 
they attended for the correct appointments. Most of the women were referred to a consultant due either to their raised BMI or another obstetric factor (eg previous caesarean section) or both. There was confusion in the reasons for referral and the women explained the consultant involvement largely due to other factors unrelated to weight.

\section{$\underline{\text { Additional tests }}$}

There was one routine intervention that many of the women found difficult; the oral glucose tolerance test (OGTT). Participants 8 and 9 described it as a "horrible test" and participant 9 questioned whether she would have it during a subsequent pregnancy. There was a lack of preparation for it for one woman:

"I had to remind my midwife about the glucose test actually and then it was almost gone past the point," (participant 4)

For others who had additional scans in later pregnancy this additional intervention became a positive experience and seen as a benefit that was not available for all women:

“..in some ways its quite nice to have the additional scans, um, it's quite reassuring to see that everything is there and, you know, because otherwise most people only have two don't they?" (participant 11)

\section{Personal responsibility}

There was clearly a desire by all participants to manage their weight and eat healthily and not one suggested that pregnancy 'allowed' them to put on weight. They spoke about watching what they ate and whilst there was a limit to what the women were encouraged to do by their midwife or obstetrician, many had a clear idea about how their behaviour could influence their weight gain:

"This time I have been a lot more sensible. I think I have kind of realised that I don't want to put the weight on again, because it's harder as you get older to actually to get rid of the weight so I think I have been a bit more sensible this time." (participant 4)

Rarer still was the proactive attitude of participant 6 who wanted to make a difference to her blood pressure. However, this might be due to the complications she had experienced in her first pregnancy due to raised blood pressure and the knowledge she had acquired due to this experience:

“..your blood pressure is an increased risk with increased weight so I was trying to walk a lot. I am just trying to reduce my blood pressure as much as possible." (participant 6)

Some women had postnatal plans to be proactive over weight loss; it appeared that being pregnant was a hiatus in their efforts to diet, exercise and control their weight 
which for some was frustrating. The women looked forward to the time after pregnancy and spoke of plans to lose weight:

"I have always been on diets and things, um, I have already got my plan and I am going back to Slimming World" (participant 9)

\section{KNOWLEDGE GAP}

This theme developed from the way the participants described their interactions with midwives and their participation in the additional tests, in particular the scans and OGTTs. Overall the women reported a lack of information about their raised BMI. There were possibly also missed opportunities for supporting the participants with their desire to manage their health and weight.

\section{Lack of information}

The majority of participants did not remember their midwives talking in any detail about their raised BMI nor why it might be important for them to know this information. An example of this comes from participant 5 :

"I got told that I had raised BMI, um and then that's as far as it went. So I haven't been told that you can have complications during your pregnancy, or your labour".

However, participant 8 recalled a good discussion with her midwife about the risks associated with her raised BMI and felt well informed. Participant 2 could also remember information about risks but she also felt that she had been given inconsistent advice which left her feeling confused and uncertain about the importance of raised BMI:

"I have been told various different things and basically that everything can be complicated by the BMI, raised BMI, but also nothing can be complicated by raised $B M I, "$

Written information, in the form of a leaflet and a sheet detailing the additional appointments recommended, was familiar to some women but not others. One participant (participant 6) said her midwife gave everything to her because she had had complications (pre eclampsia) in her last pregnancy. Another said that nothing had been explained to her (participant 4). Participant 3 found the written care pathway for women with a raised BMl useful as a way of knowing what appointments she had ahead of her. However, she too, explained that she was not given reasons for the additional appointments.

\section{Past experience}

Those participants who had had previous pregnancies often drew on this experience and felt that they were more knowledgeable about raised BMI and pregnancy because of it. This was not universally true: 
"I mean it would have perhaps been useful to know what those tests were going to be in the future of the pregnancy, um, and you know, what the difference was going to be between the first pregnancy and the second one." (participant 9)

There was also a belief that their previous experiences were a reason why their midwife in their current pregnancy did not discuss their BMI in detail.

"I think after having had the two children already they probably thought, you know, perhaps they don't need to go into too much detail." (participant 3)

There were also other obstetric reasons why women had higher risks, including previous caesarean section, which involved a consultant appointment. For women this appeared to be the primary reason for antenatal surveillance (scans and extra appointments) and raised BMI was less significant. Even when a participant had extra scans or appointments it was not always clear why:

"..because I am under consultant care I have been told by one midwife that it's because of my BMI mainly whereas the other midwife I have been seeing recently said 'no' it's to do with my daughter's birth weight," (participant 2)

Communication around the scan

The first trimester scan report became a concern to some women due to its wording:

"..on my scan notes um even though they have um a perfect view of and managed to see everything they still put on your notes 'restricted view due to raised BMI'." (participant 1)

This certainly was not a concern for all participants, but those questioned felt that the caveat about restricted view was written for all women with a raised BMI whether or not the view had been restricted. They felt it was a tick box exercise (participant 10) or one of "covering their backs" (participant 1).

Need for information

Participants reported a lack of discussion about exercise and diet. "I think she just found it a little bit awkward and didn't really feel like talking about it, but then again I didn't ask questions about it either." (participant 3)

There was a desire for more information from some women. Discussion about the woman's diet was mostly confined to what is best omitted in the diet in pregnancy. However, more information would have been helpful to some women:

"I think it would be nice, um, again to talk about diet or them to ask you what you are eating or what you should be doing, should you be doing exercise or asking you, and maybe it should be a question of 'do you exercise' or 'what do you do, what do you eat?"'(participant 5) 
"I think you do need to know [about raised BMI], and it can't be sugar coated" (participant 8)

One participant (2) did report that her midwife had told her to go on a diet but this was the exception. Another felt that her midwife could add nothing to what she knew about healthy eating:

"..because I know that I have got bigger, or whatever, so you know there is nothing they should have said, or I could have asked.." (participant 7)

There was also a lack of information about exercising in pregnancy. Three of the women suffered from pelvic girdle pain and were restricted in their daily activities. One, who said she was usually active, explained that he didn't actually feel like doing much exercise towards the end of the pregnancy. Only three women spoke about exercising as important in pregnancy. The quote below encapsulates the benefits extremely well:

"..but I think definitely exercise is important, because not only would it benefit you, you know physically, um, it gives stress relief and also there is everything about keeping active makes labour easier." (participant 8)

Others were asked what they felt their midwives could offer in terms of additional support. A support group of women with raised BMls was one suggestion (participant 8) whilst another suggested a postnatal walking group (participant 9)

Not all participants thought a postnatal support group would benefit them but thought that additional information from their midwife would be helpful about where to go for groups and classes (participant 10).

Time was an issue as well to some participants:

"So it's just how to juggle, it's not really the losing of the weight, because everyone knows how to do that, but it's how to juggle children and losing the weight." (participant 9)

Another area that some women felt they would like addressed was how much weight they should safely gain in pregnancy:

"..there has not been any discussion. There has been no discussion at all about my weight, um, whether being overweight, or you know, the amount of weight I have put on in pregnancy there hasn't been any, no discussion about it at all." (participant 4)

\section{DISCUSSION:}

The findings describe how women experience being pregnant with a raised BMI. Qualitative methodology is not intended to be representative of the broader population but rather gain a deeper understanding of this group of women. 
Some women in this study felt judged and criticised by health professionals and this has been found in other studies (Furber \& McGowan 2011a; Smith \& Lavender 2011). However, this was not universal and most women reported a supportive relationship with their midwife although not all felt that their midwife dealt with the issue of their raised BMI without embarrassment. The women were largely acutely aware of their raised $\mathrm{BMI}$ and sensitive to the words and actions of the health professionals they saw.

The findings demonstrated that the women interviewed lacked knowledge about raised $\mathrm{BMI}$ and risk as well as the reasons for additional interventions and this adds to findings from other research (Shub et al. 2013; Heslehurst 2014). This gap in knowledge could be explained by midwives not communicating this information (time pressure, knowledge, embarrassment) or the women forgetting the discussions or a combination of both. What is of note is that there was a real lack of understanding by some of the women why their particular care pathway was necessary. There is evidence to demonstrate that midwives are lacking in skills and confidence to support women with a raised BMI (Furness et al. 2011; Stotland et al. 2010; Schmied et al. 2011) which can express itself as embarrassment. Another suggestion from a qualitative study (Foster \& Hirst 2014) was that midwives' own body images influenced how and if they approached discussions on BMI. A systematic review (Heslehurst et al. 2014) found evidence about the lack of training for midwives and the difficulties they found in communicating with women about raised BMI.

Women in a study by Lavender et al (2014) reported that they often did not have a discussion with midwife about weight and lifestyle despite being ready to make lifestyle changes. In a separate study, women knew it was important to eat well and be physically active but lacked the "practical application of knowledge" (de Jersey et al. 2013). The study found that advice from midwives beyond encouragement was limited. This is supported by the findings of a study by McParlin et al (McParlin et al. 2017) which found midwives understood physical activity to be an important part of their job but lacked the skills and resources and did not prioritise these discussions.

The terminology used by midwives was consistent in this study and 'raised BMl' was considered acceptable and appropriate. This is in contrast to the finding of another study (Schmied et al. 2011) in which women felt that raised BMI was a euphemism behind which midwives hid to avoid embarrassment. An interesting finding of this study is that the women did not consider themselves obese although all but one recognised that they were overweight. The word 'obese' was clearly unacceptable and a negative term and this has been found in other studies (Gray et al. 2011; Volger et al. 2012). This may be an area to further consider in the context of NHS health care as 'obese' is commonly used as a clinical term but appears to have little relevance to the group of pregnant women in this study.

The women interviewed explained that they were keen to follow guidelines and recommendations. They did not use pregnancy as a reason to over eat and were 
conscious about their diet. Some displayed vigilance over their weight with some weighing themselves frequently throughout pregnancy. There are, however, no UK guidelines about healthy weight gain in pregnancy (NICE 2010). Although most women were thinking about their diet and weight they did not have any professional guidelines about what they should aim for. Many of these women would be used to target weights from their diet clubs and this may have been helpful for them. A different finding was seen in an earlier study (Weir et al. 2010) which investigated physical activity and found that women were relatively unconcerned about weight gain although they were aware of healthy behaviours.

With regard to eating patterns, women in another qualitative study (Paterson et al. 2016) experienced satiety more readily in pregnancy and it is suggested that this could be a "teachable moment" around satiety and responding to body cues. The opportunity for behaviour change in pregnancy has also been identified in other studies (Dinsdale et al. 2016; Mills et al. 2011; Dencker et al. 2016). Another finding from one of the studies (Dencker et al. 2016) was that women who were found to have a good knowledge of raised BMl and risk, but whilst this motivated them it also made them anxious.

Two recent trials have introduced lifestyle interventions for pregnant women with a raised BMI. The LIMIT randomised trial (Dodd et al. 2014) in Australia reported that there was no significant difference between the intervention and control groups in the risk of the baby being large for gestational age, nor in maternal pregnancy and birth outcomes (although babies born to women in the lifestyle advice group were significantly less likely to have birth weight above $4000 \mathrm{~g}$ ). The UPBEAT study (Poston et al. 2015) from the UK found that the primary outcomes (gestational diabetes and babies being large for gestational age) did not differ between the intervention and control groups. However there were improvements in maternal weight gain and increased physical activity in the intervention group.

These two important trials appear to have shown little difference in primary outcome between a lifestyle intervention and usual care. The obesogenic environment and social context in which the woman and her family live are clearly important influences (Dencker et al. 2016). Another consideration regarding lifestyle changes comes from the findings of a recent study which showed that caution is needed with weight loss fluctuations between pregnancies, as women might be a risk of having a small for gestational age baby with inter-pregnancy weight loss (Wallace et al. 2017).

The women of this study were not forthcoming about what additional support could be given by midwives in terms of their BMI and pregnancy. There was little consideration of physical activity and healthy lifestyles were mainly considered in terms of food and diets. The postnatal period was suggested by one participant as a time to address her weight, which was one of the findings of a meta-synthesis (Smith \& Lavender 2011). However, another participant suggested that the postnatal time is 
a busy one of juggling family life and not conducive to focusing on weight management.

One way of helping to discuss with women the risks associated with a raised BMI would be to use high quality information explaining what this means for them and the rationale behind recommended tests. A recent systematic review from Cochrane on using decision aids (Stacey et al. 2017) supports the use of decision aids as they improve patients' knowledge of the options and feel better informed and more clear about what matters most to them. Such collaborative decision making could help women to make better informed choices about their care.

\section{Strengths and limitations of this study}

The fact that a midwife was interviewing the women could have influenced some of the discussions with a power imbalance between interviewer and interviewee.

\section{Conclusions}

The findings suggest that pregnant women with a raised BMI need clear information, perhaps regularly given, by midwives who have been trained in both the value of healthy eating and physical exercise as well as how to communicate this. Recent trials into interventions have not demonstrated that antenatal lifestyle advice helps to reduce gestational diabetes or large for gestational age babies. However, there is a demand for support for this group of women and their psychological needs should also be central to their care. 


\section{References}

Amir, L.H. \& Donath, S., 2007. A systematic review of maternal obesity and breastfeeding intention, initiation and duration. BMC pregnancy and childbirth, 7(1), p.9. Available at: http://www.biomedcentral.com/1471-2393/7/9 [Accessed October 11, 2014].

Bayrampour, H. et al., 2012. Advanced maternal age and risk perception: a qualitative study. BMC pregnancy and childbirth, 12(1), p.100. Available at: http://www.biomedcentral.com/1471-2393/12/100 [Accessed September 11, 2014].

Carlson, N.S. \& Lowe, N.K., 2014. Intrapartum Management Associated with Obesity in Nulliparous Women. Journal of Midwifery \& Women's Health, 59(1), pp.4353. Available at: http://dx.doi.org/10.1111/jmwh.12073.

Carryer, J., 2001. Embodied largeness: a significant women's health issue. Nursing Inquiry, 8(2), pp.90-97.

Dencker, A. et al., 2016. Adopting a healthy lifestyle when pregnant and obese - an interview study three years after childbirth. BMC Pregnancy And Childbirth, 16(1), p.201. Available at: http://search.ebscohost.com/login.aspx?direct=true\&db=cmedm\&AN=27473076 \&site=ehost-live.

Dinsdale, S. et al., 2016. "As soon as you 've had the baby that 's it ..." a qualitative study of 24 postnatal women on their experience of maternal obesity care pathways. BMC Public Health, pp.1-13. Available at: http://dx.doi.org/10.1186/s12889-016-3289-1.

Dodd, J.M. et al., 2014. Antenatal lifestyle advice for women who are overweight or obese: LIMIT randomised trial. BMJ (Clinical research ed.), 348(February), p.g1285. Available at: http://www.pubmedcentral.nih.gov/articlerender.fcgi?artid=3919179\&tool=pmcen trez\&rendertype=abstract [Accessed October 21, 2014].

Eley, V.A. et al., 2014. The effect of antenatal anaesthetic consultation on maternal decision-making, anxiety level and risk perception in obese pregnant women. International Journal of Obstetric Anesthesia, 23(2), pp.118-124. Available at: http://www.sciencedirect.com/science/article/pii/S0959289X13001556.

Foster, E. \& Hirst, J., 2014. Midwives' attitudes towards giving weight-related advice to obese pregnant women. British Journal of Midwifery, 22(4).

Furber, C.M. \& McGowan, L., 2011a. A qualitative study of the experiences of women who are obese and pregnant in the UK. Midwifery, 27(4), pp.437-444. Available at: http://www.sciencedirect.com/science/article/pii/S0266613810000562.

Furber, C.M. \& McGowan, L., 2011b. A qualitative study of the experiences of women who are obese and pregnant in the UK. Midwifery, 27(4), pp.437-44. 
Available at: http://www.ncbi.nlm.nih.gov/pubmed/20483513 [Accessed September 30, 2014].

Furness, P.J. et al., 2011. Maternal obesity support services : a qualitative study of the perspectives of women and midwives. BMC Pregnancy and Childbirth, 11(1), p.69. Available at: http://www.biomedcentral.com/1471-2393/11/69.

Gray, B.A., 2006. Hospitalization history and differences in self-rated pregnancy risk. Western journal of nursing research, 28(2), pp.216-229.

Gray, C.M. et al., 2011. Words matter : a qualitative investigation of which weight status terms are acceptable and motivate weight loss when used by health professionals.

Heslehurst, N., 2014. Implementation of pregnancy weight management and obesity guidelines : A meta- synthesis of healthcare ... Obesity Management / Pregnancy Implementation of pregnancy weight management and obesity guidelines : a meta-synthesis of healthcare professionals '. , (April).

Heslehurst, N. et al., 2014. Interventions to change maternity healthcare professionals $i$ behaviours to promote weight-related support for obese pregnant women: a systematic review. Implementation science: IS, 9(1), p.97. Available at: http://www.implementationscience.com/content/9/1/97 [Accessed November 22, 2014].

Heslehurst, N. et al., 2007. Obesity in pregnancy: a study of the impact of maternal obesity on NHS maternity services. BJOG: An International Journal of Obstetrics \& Gynaecology, 114(3), pp.334-342. Available at:

http://dx.doi.org/10.1111/j.1471-0528.2006.01230.x.

de Jersey, S.J. et al., 2013. An observational study of nutrition and physical activity behaviours, knowledge, and advice in pregnancy. BMC Pregnancy and Childbirth, 13(1), p.1. Available at: BMC Pregnancy and Childbirth.

Krishnamoorthy, U., Schram, C.M.H. \& Hill, S.R., 2006. Maternal obesity in pregnancy: Is it time for meaningful research to inform preventive and management strategies? BJOG : an international journal of obstetrics and gynaecology, 113(10), pp.1134-40. Available at:

http://www.ncbi.nlm.nih.gov/pubmed/16972858 [Accessed October 9, 2014].

Lavender, T. et al., 2001. Midwives' views on redefining midwifery 1: health promotion. British Journal of Midwifery, 9(11), pp.666-670. Available at: http://dx.doi.org/10.12968/bjom.2001.9.11.9409.

McParlin, C. et al., 2017. What helps or hinders midwives to implement physical activity guidelines for obese pregnant women? A questionnaire survey using the Theoretical Domains Framework. Midwifery. Available at: http://dx.doi.org/10.1016/j.midw.2016.09.015.

Mills, A., Schmied, V. \& Dahlen, H., 2011. â€ $€^{T M}$ Get alongside us ', women â€ ${ }^{T M} s$ experiences of being overweight and pregnant in Sydney, Original Article overweight and pregnant in Sydney, Australia. , (December). 
Molyneaux, E. et al., 2014. Obesity and mental disorders during pregnancy and postpartum: a systematic review and meta-analysis. Obstetrics and gynecology, 123(4), pp.857-867.

NICE, 2007. Antenatal and postnatal mental health. Clinical guideline 45.

NICE, 2010. Weight management before, during and after pregnancy | Guidance and guidelines |PH27 NICE. Available at:

https://www.nice.org.uk/guidance/ph27\#.WXw951Ad1GU.mendeley [Accessed July 29, 2017].

Paterson, H., Hay-Smith, E.J.C. \& Treharne, G.J., 2016. Women's experiences of changes in eating during pregnancy: A qualitative study in Dunedin, New Zealand. New Zealand College of Midwives Journal, (52), pp.5-11. Available at: http://search.ebscohost.com/login. aspx?direct=true \&db=c8h\&AN=114938709\&si te=ehost-live.

Poston, L. et al., 2015. Eff ect of a behavioural intervention in obese pregnant women ( the UPBEAT study ): a multicentre , randomised controlled trial. , pp.767-777.

Schmied, V. a et al., 2011. "Not waving but drowning": a study of the experiences and concerns of midwives and other health professionals caring for obese childbearing women. Midwifery, 27(4), pp.424-30. Available at: http://www.ncbi.nlm.nih.gov/pubmed/20381222 [Accessed October 9, 2014].

Shub, A. et al., 2013. Pregnant women 's knowledge of weight, weight gain , complications of obesity and weight management strategies in pregnancy. BMC Research Notes, 6(1), p.1. Available at: BMC Research Notes.

Smith, D. \& Lavender, T., 2011. The maternity experience for women with a body mass index $\geq 30 \mathrm{~kg} / \mathrm{m} 2$ : a meta-synthesis. BJOG: An International Journal of Obstetrics \& Gynaecology, 118(7), pp.779-789. Available at: http://dx.doi.org/10.1111/j.1471-0528.2011.02924.x.

Stacey, D. et al., 2017. Decision aids for people facing health treatment or screening decisions ( Review ) Decision aids for people facing health treatment or screening decisions., (4).

Stotland, N.E. et al., 2010. Preventing excessive weight gain in pregnancy: how do prenatal care providers approach counseling? Journal of Women's Health (15409996), 19(4), pp.807-814. Available at:

http://search.ebscohost.com/login.aspx?direct=true\&AuthType=ip,url,shib\&db=rz h\&AN=2010644680\&site=ehost-live.

Volger, S. et al., 2012. Patients' Preferred Terms for Describing Their Excess Weight: Discussing Obesity in Clinical Practice. Obesity, 20(1), pp.147-150. Available at: http:https://dx.doi.org/10.1038/oby.2011.217.

Wallace, J.M., Bhattacharya, S. \& Horgan, G.W., 2017. Weight change across the start of three consecutive pregnancies and the risk of maternal morbidity and SGA birth at the second and third pregnancy. , pp.1-15. 
Weir, Z. et al., 2010. Physical activity in pregnancy: a qualitative study of the beliefs of overweight and obese pregnant women. BMC Pregnancy and Childbirth, 10(1), p.18. Available at: http://dx.doi.org/10.1186/1471-2393-10-18.

Wray, S. \& Deery, R., 2008. The Medicatlization of Body Size and Women's Healthcare. Health Care for Women International, 29, pp.227-243.

Yu, C.K.H., Teoh, T.G. \& Robinson, S., 2006. Obesity in pregnancy. BJOG : an international journal of obstetrics and gynaecology, 113(10), pp.1117-25. Available at: http://www.ncbi.nlm.nih.gov/pubmed/16903839 [Accessed October 11, 2014]. 